\title{
On-line adaptive side-by-side human robot companion in dynamic urban environments
}

\author{
Ely Repiso $^{\dagger} \quad$ Gonzalo Ferrer ${ }^{\ddagger} \quad$ Alberto Sanfeliu ${ }^{\dagger}$
}

\begin{abstract}
This paper presents an adaptive side-by-side human-robot companion approach for navigation in urban dynamic environments, based on the anticipative kinodynamic planning. The adaptive means that the robot is capable of adjusting its motion to the behavior of the person being accompanied. Our main objective is to optimize in real time the path performed by the pair human-robot, by modifying dynamically the angle and distance between both throughout different locations of the path. We have defined a new cost function for finding the best planned path that takes into account the cost of the geometrical configuration between the human and the robot. Moreover, we have modified the Extended Social Force Model (SFM) to include the required forces to maintain the angle and distance between the robot and human while the human-robot pair is moving towards the shared goal. The method has been validated throughout a large set of simulations and real-live experiments.
\end{abstract}

\section{INTRODUCTION}

In the future, we expect to see social robots sharing urban areas with people. To achieve that, robots have to develop several skills such as navigating along with humans. There are many situations where robots have to develop the accompany task: guiding people in museums [1], shopping in malls [2], following people and learning objects and places to future interactions [3], helping aging people as home-care robots to live independently [4], accompanying a person to some place while walking in side-by-side formation [5], or accompanying groups of people [6].

In urban environments, people tend to walk in groups while avoiding other people and obstacles, they usually walk side-by-side or close to this configuration (in front or behind) depending on the environment.

Human-robot side-by-side navigation is a challenging task because the robot has to navigate in a safe and natural way while accompanying a person. Also, the robot has to adapt its behaviour to avoid collisions with obstacles while not disturbing other people in the environment. An example of this is shown in Fig. 1, where a robot maintains a formation, and adapts its motion while navigates among obstacles. Also, this behavior is a complex task for robots because requires

Work supported by the Spanish Ministry of Science and Innovation under project ROBOT-INT-COOP(DPI2013-42458-P).

${ }^{\dagger}$ The authors are with the Institut de Robòtica i Informàtica Industrial (CSIC-UPC). Llorens Artigas 4-6, 08028 Barcelona, Spain. \{erepiso, sanfeliu\}@iri.upc.edu

$\ddagger$ The author is associated with the University of Michigan, Ann Arbor. gferrermeumich.edu

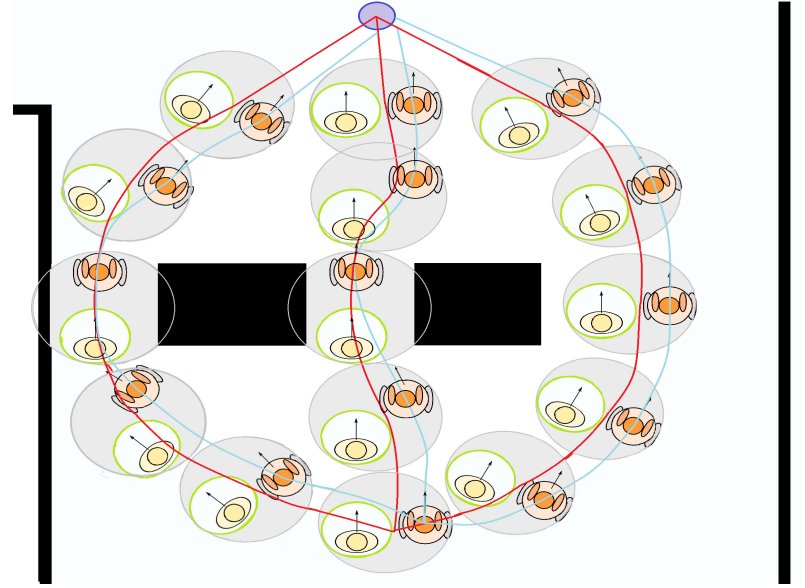

Fig. 1. It can be seen three solution paths for the human-robot side-by-side navigation. The second path is the best one, the shorter one, although the geometrical configuration between the human and the robot is not side-byside.

several robotic cognitive functions as perception, prediction, navigation and human-robot interaction.

In this paper, we propose a modification of the Anticipate Kinodynamic Planning (AKP) [7], [8] that includes a new model to find the optimal geometrical configuration between the human and the robot (distance and angle between them) at each discrete time of the path to afford the narrow passageways to arrive to the goal in the minimum time. In this new model, we propose a new cost function (denominated companion cost) that takes into account the cost of modifying the angle and distance between the human and robot to afford the dynamic and static passageways and a reformulation of the Extended Social Force Model [9], [6]) to include the new force that maintains the side-by-side configuration while navigating. These new cost and new force are computed taking into account that the robot always has to adapt to the person's trajectory decisions.

In the following sections, first the related work is displayed. Then, we explain how we improved the Anticipative Kinodynamic Planner (AKP) including the navigation of the pair robot-human. In Sec. III, we introduce a new cost function, denominated companion cost, to take into account the effect of the diverse geometrical configurations between the robot and the person (distance and angle between them to walk side-by-side) in the computation of the best path. In Sec. IV, we extend the Extended Social Force Model (ESFM) using a new force that takes into account the navigation of 
the pair robot-human. In Sec. V we explain the results in the simulations and finally, in Sec. VI, we show the results in real-live experiments.

\section{RELATED WORK}

Human-robot side-by-side navigation research is relatively new in comparison to traditional robot navigation, where robots navigate in a safe and human like manner. However, human-robot companion algorithms are increasingly evolving into safer and more realistic behaviours.

As a starting point, some researchers developed methods which use reactive approaches for robots to accompany people. Prassler et al. [10] perform a reactive companion behavior for a mobile wheelchair robot. Sviestins et al. [11] study the relative position and walking speed of people while walking with a robot. They estimate the preferred speed of the companion person allowing the robot to adapt its speed while both walk side-by-side, but their method did not consider walking in a dynamic environment. In our work, we consider not only static, but also dynamic narrow passageways while the pair human-robot walks side-by-side.

Since reactive approaches are not suited to obtain a realistic and safe companion task, some studies developed methods that include some prediction to anticipate the behaviour of the partner and, thus, to obtain a more intelligent navigation. Murakami et al. [12] perform and compare two approaches for walking side-by-side: the leader-follower and the collaborative. In the collaborative approach, they make a velocity based prediction method where both group members know the goal. In the leader-follower, the robot does not know the goal and follows the person by estimating the local destination from the person direction. They finally conclude that the leader-follower approach incorporates more advantages than the other. Morales et al. [13], [14] proposed a model which describes people walking side-by-side and used this model to predict the future next position of the partner and to plan the next robot position. In our approach, we also predict the future next position, but we also look for the best path to not bother the human group trajectories and do the best path in the minimum time, moreover, we take into account the dynamic narrow passageways.

Finally, Kuderer et al. [15] explored the possibility to face the problem using reinforcement learning. They apply reinforcement learning to teach a tele-operated robot how to navigate in a cooperative way avoiding collisions with obstacles.

In contrast to previous studies that only considered navigating near the person in a fixed side-by-side formation, our method allows a dynamic positioning around the human partner facilitating the navigation inside the group and minimizing the effort to avoid people in the dynamic environment. This dynamic positioning around the human allows to deal with cases where it is better to break momentarily the sideby-side formation to navigate in a joint, as humans do in a natural way.Furthermore, the presented approach selects the path that allows the group to walk in side-by-side formation, but if the human prefers to go through a narrow passage the robot reformulates its path and goes through the passage accompanying the person. Moreover, most of the previous approaches only take into account one step prediction. In contrary, our approach predicts in real time the dynamic movement of the partner and the rest of people in a horizon time [16] (the horizon time sets the amount of time that the planner forecasts the movement of all the dynamic elements) including the static obstacles. This horizon time allows the robot to predict the next movements of people and it is able to anticipate their navigation behaviours. Works on people tracking are numerous, we follow the approach of Vaquero et al. [17].

\section{GLOBAL HUMAN-ROBOT COMPANION NAVIGATION}

As we have discussed previously, the goal of this work is to make robots capable of navigating side-by-side (see Fig. 1), although they can modify the geometrical configuration (see Fig. 3-right) in diverse parts of the path due to narrow passageways. These narrow passageways can be formed by static obstacles of the infrastructure or by people walking in front of the couple human-robot, and in this case, the narrow passageways will be created dynamically. In any of these cases the computation of the best navigation sideby-side has to be done on-line and in real time. In this section, we will explain a modification of the Anticipate Kinodynamic Planning explained in [8] which includes a new cost function that takes into account the cost of walking depending on the configuration. This cost function is added to the cost functions defined in the AKP method [8], which includes the cost of the distance until the destination, the robot orientation to the destination, the robot control and the costs obtained from the objects and other persons in the environment. These cost functions are used to select the best path between each one of the steps in the computation of the RRT planner [18] for obtaining the best path to destination.

When the couple human-robot is navigating in a narrow passageway they can dynamically change their configuration. The robot can select to go in front of the person, if the person reduces its speed, or vice versa, the person can select going in front of the formation. In both cases, changing the configuration implies an additional cost that has to be considered.

Before explaining the new cost function, let us define the parameters and variables of the geometrical configuration of the pair human-robot, see Fig. 3. $R_{e}$ is the radius of the circle containing the inflated robot (radius $R_{r}$ ) and person $\left(R_{p}\right)$, and the minimum distance between the center of the person and the robot, $2 R_{i}$. Below, the formulas that relate these parameters and variables are shown.

$$
\begin{gathered}
R_{e}=R_{i}+\max \left(R_{r}, R_{p}\right) \\
R_{i} \geq\left(R_{r}+R_{p}\right) / 2
\end{gathered}
$$

Now, let us compute the best angle between the person and the robot (of the pair) for each point of the path, to navigate while avoiding static and dynamic obstacles. Let us define $d_{o}$, Eq. (3), as the minimum distance between the center of 


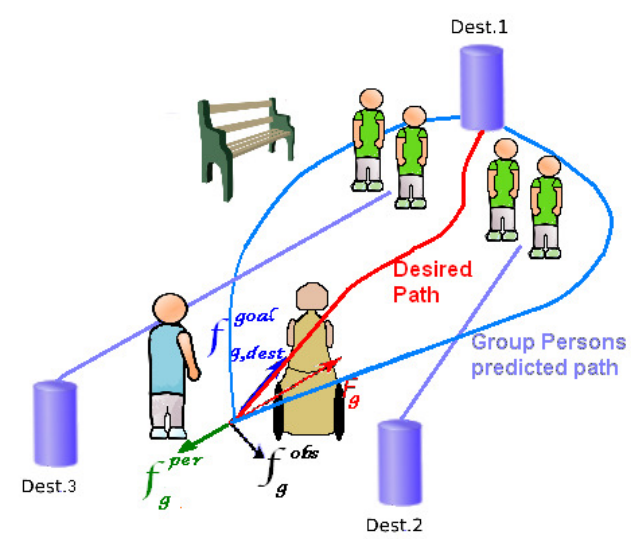

Fig. 2. A pair robot-human going to a destination is shown, while two groups of people are moving in the opposite direction. Three alternative and valid paths are plotted. The red path is the best path to go to the destination and the blue paths are the discarded paths, due to their higher cost. In reference to the forces, the summation of all the forces applied to the center of the pair is the red arrow $F_{g}$. The blue arrow represents the attractive force to the destination, $\mathbf{f}_{g \text {,dest }}^{\text {goal }}$. The green and black arrows represent the different interaction forces between the pair and other people $\mathbf{F}_{g}^{p e r}$ and obstacles $\mathbf{F}_{g}{ }^{\text {obs }}$
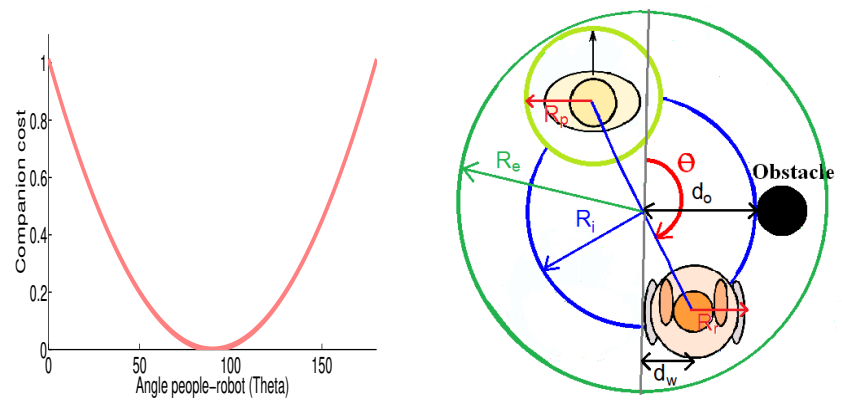

Fig. 3. The right figure shows the cost companion graph and the left figure shows the computation of the companion angle for a possible obstacle collision.

the pair and the nearest obstacle. Also, $2 R_{i}$ is the distance between the center of the person position and the center of the robot position and the value of $R_{i}$ is advisable to be $R_{i} \geq$ $0.75 \mathrm{~m}$. Let's remark that its $R_{i}$ includes the $\max \left(R_{r}, R_{p}\right)$ and one half of the real distance between person and robot. Then, we can obtain the best angle between the person and the robot, $\theta$ of Fig. 3, to navigate while avoiding obstacles, using the Eq. (5). To calculate potential collisions we use a predefined map and laser scans. Also, these two were used to localize the robot inside the environment.

$$
\begin{gathered}
d_{o}>d_{w}+\max \left(R_{r}, R_{p}\right) \\
d_{w}=R_{i} \sin (\pi-\theta) \\
\theta=\pi-\arcsin \left(\frac{d_{w}}{R_{i}}\right), \text { for } \theta \in\left(-\frac{\pi}{2}, \frac{\pi}{2}\right]
\end{gathered}
$$

The term $d_{w}$ is the shortest distance between the robot center and the direction of movement of the group. The new cost function is based on $\theta$. When the pair humanrobot is navigating side-by-side, the minimum cost is for $\theta=\pi / 2$, and any other angle implies a cost of navigation. The worst cost case is for $\theta=\pi$, when the robot (person) is in front the person (robot). The pair robot-human will try to navigate keeping the side-by-side formation, but sometimes it is needed to obtain a worst companion cost to pass through a narrow passage or avoid different groups of people. One example of this situations can be seen in the Fig. 1. Furthermore, if the pair robot-human has to chose between a path where they can walk side-by-side or a path where they have to breach this formation, the couple will select the path that has the minimum cost. The new cost $J_{c}$, denominated companion cost, is defined as follows:

$$
J_{c}(S)=\eta\left\|\theta(t)-\theta^{0}\right\|^{2},
$$

where $S$ is the state of the robot and all the people, which includes position, velocity and time and additionally orientation for the robot[7]. The $\eta$ is a normalization parameter, taking into account that $\theta(t) \in[0-\pi]$ and we want to obtain a maximum value of 1 around $\pi / 2, \theta(t)$ is the needed angle between person and robot in each position of the path at time $t$, and $\theta^{0}$ is the best companion angle, which is $\pi / 2$ degrees in our case, to allow the walking side-by-side. Fig. 3-left plots the companion cost of Eq. (6).

This new companion cost, $J_{c}$ is added to the cost functions defined in the AKP method as was previously commented. Then the new cost function $\mathbf{J}\left(S, s_{\text {goal }}, U\right)$ will contain all the associated costs, that is: $\mathbf{J}\left(S, s_{\text {goal }}, U\right)=$ $\left[J_{d}, J_{o r}, J_{r}, J_{p}, J_{o}, J_{c}\right]$. These cost functions are described in [8] and Eq. (6). Then, the multi-objective cost function becomes a single-objective cost function by applying a three step calculation. First, we obtain each individual cost function. Second, to avoid the scaling effect of weighted-sum method, we normalize each cost function to $(-1,1)$, according to the equation:

$$
\bar{J}_{i}(\mathbf{X})=\operatorname{erf}\left(\frac{x-\mu_{x}}{\sigma_{x}}\right)
$$

where the variables $\mu_{x}$ and $\sigma_{x}$ are estimated after the tree of the RRT's possible planning paths are computed. And finally, we calculate a projection via weighted sum $J: R^{I} \rightarrow R$ as follows and we obtain the final weighted cost formula (see [8] for additional explanation):

$$
J\left(S, s_{\text {goal }}, U\right)=\sum_{i} w_{i} \bar{J}_{i}\left(S, s_{\text {goal }}, U\right) .
$$

In our dynamic planning we minimize the multiple cost functions and we use the same computation process described in [8]. With respect to the weights, we use the same procedure described in that paper, but for the simulations and real-live experiments we have used the following weights: $5 / 6$ for the previous costs, because they consider 5 navigation aspects and $1 / 6$ for the new companion cost. Finally, for each time $t$, we calculate the different solution paths, where each of these paths have a final cost that includes the companion cost, and the robot selects the path with the minimum cost. An example of several path calculation for each iteration time is shown in the Fig. 2.

\section{EXTENSION OF THE SOCIAL FORCE MODEL}

So far, we have computed the best path for the pair humanrobot, which is obtained with the extension of the AKP, presented previously in Sec. III. Then, we have to control the robot movement by computing the force $\mathbf{F}_{r}$ that has 
to be applied in order to follow the best path. This force is computed as follows using the Extended Social Force Model:

$$
\begin{gathered}
\mathbf{F}_{r}=\alpha \mathbf{f}_{r, p}^{\text {goal }}\left(\mathcal{D}_{n}\right)+\left(\gamma \mathbf{F}_{r}{ }^{p e r}+\delta \mathbf{F}_{r}{ }^{\text {obs }}\right) \\
\alpha=1-\gamma-\delta
\end{gathered}
$$

where, $\gamma$ and $\delta$ were obtained as described in [5].

The attractive force assuming that the robot $r$ tries to adapt its velocity within a relaxation time $k^{-1}$, but taking into account at the same time the distance and angle of the accompany person to destination $\left(\mathcal{D}_{n}\right)$, is computed as follows:

$$
\mathbf{f}_{r, p}^{\text {goal }}\left(\mathcal{D}_{n}\right)=k\left(\mathbf{v}_{r}^{0}\left(\mathcal{D}_{n}\right)-\mathbf{v}_{r}\right)
$$

Where $\mathbf{v}_{r}^{0}\left(\mathcal{D}_{n}\right)$ is the desired velocity vector to reach the goal according to the destination $\mathcal{D}_{n}$ and $\mathbf{v}_{r}$ is the current velocity of the robot. This is the force that keeps the robot in the computed geometrical configuration (robot-human) to allow the joint navigation.

Equation 11 is applied at each discrete point of the path to reach the goal. Let us consider that there are $t=1,2, \ldots, M$ discrete points in the path, then the attractive force of the next discrete point $\mathbf{f}_{r, p}^{\text {goal }}(t+1)$ will be computed as:

$$
\mathbf{f}_{r, p}^{\text {goal }}(t+1)=k\left(\mathbf{v}_{r}^{0}\left(P_{r}(t+1)\right)-\mathbf{v}_{r}\left(P_{r}(t)\right)\right.
$$

where $P_{r}(t+1)$ is the next position of the robot taken into account the constraints in distance and angle imposed by the person (see Fig. 3-right) and $P_{r}(t)$ is the current position of the robot. Because the robot has always to follow the person and there are constraints in distance and angle between the robot and the person, the computation of the next position of the robot, $P_{r}(t+1)$, will depend on the next position of the person, $P_{p}(t+1)$. If we consider the two components of the position of the robot, $P_{r}(t+1)=\left(X_{r}(t+1), Y_{r}(t+1)\right)$ and of the position of the person, $P_{p}(t+1)=\left(X_{p}(t+1), Y_{p}(t+1)\right)$, then, the computation of $P_{r}(t+1)$ will be:

$$
\begin{array}{r}
\hat{x}_{r}(t+1)=\hat{x}_{p}(t+1)+2 R_{i} \cos \left(\theta_{p}-\operatorname{sgn}\left(\theta_{p}-\theta_{c}\right) \theta_{i}\right) \\
\hat{y}_{r}(t+1)=\hat{y}_{p}(t+1)+2 R_{i} \sin \left(\theta_{p}-\operatorname{sgn}\left(\theta_{p}-\theta_{c}\right) \theta\right)
\end{array}
$$

where, $2 R_{i}$ is the distance between the robot and the person center positions. $\theta_{p}$ is the person orientation to the destination and is obtained with the Eq. (15). Then, $\theta_{c}$ is the real companion angle between the person and robot positions. Finally, $\theta$ is the ideal companion angle between the robot and the person, which takes into account the person position and orientation of movement and is calculated with the Eq. (5). $R_{i}, \theta_{p}, \theta_{c}$ and $\theta$ are computed in the discrete time $t$. These equations are meant to place the robot in one of the sides of the person taking into account the proximity of the robot to one of the person sides.

$$
\theta_{p}=\operatorname{atan}\left(\frac{Y_{\text {goal }}-Y_{p}}{X_{\text {goal }}-X_{p}}\right)
$$

where, $\left(X_{p}, Y_{p}\right)$ is the person position and $\left(X_{\text {goal }}, Y_{\text {goal }}\right)$ is the goal position which is selected amount all the possible goals of the environment taking into account the companion person prediction [16], both in discrete time $t$. In case that the final destination is not know, the robot predicts few meters in advance the person path to accompany her/him.
The repulsive force respect to the other pedestrians in the dynamic environment is:

$$
\mathbf{F}_{r}^{p e r}=\sum_{j \in P} \mathbf{f}_{r, j}^{i n t}
$$

The repulsive force respect to the obstacles in the environment is:

$$
\mathbf{F}_{r}^{o b s}=\sum_{o \in O} \mathbf{f}_{r, o}^{i n t}
$$

These repulsive forces are used by the robot to avoid possible collisions or unnecessary interactions with other pedestrians (see Ferrer et al. [5]).

\section{SIMULATIONS}

We have first evaluated the model in simulation, in order to test controlled situations that may occur. We have carried out 2126 simulations of the companion task for the robot in presence of obstacles and other people. Our simulated robot is modelled as a non-holonomic vehicle, as the real robot. Also, in all the experiments we use $\eta=1.2346 e^{-4}$ to normalize the equation 6 . We have used the people simulator which uses the same extended social force model from [7] and we have randomly changed the velocity for all the people between $[0-1] \mathrm{m} / \mathrm{s}$. To test the algorithm, we have made three experimental settings. The first consists of a situation where the robot has to accompany the person to a destination and the couple has to pass through a static narrow passageway (Fig. 6). In the second one, the robot has to accompany a person to a destination and the couple crosses two other people groups that forms a dynamic narrow passageway, where the couple has to pass through (Fig. 7). The third experiment combines both cases, where the couple has to avoid obstacles and people at the same time (Fig. 8). In each environment the robot is able to overcome the obstacles walking in front or behind of the companion person. Furthermore, the best companion angle for the task was changed to $5 \pi / 9$ to get closer to the real experiments, where the robot has a small laser gap in the side-by-side position of $\pi / 2$.

The metric to evaluate the companion task for the robot is plotted in Fig. 4. For the performance in distance, we have considered that the robot makes a good performance if keeps its position inside of the following interval: $[0.75-2] \mathrm{m}$. The horizontal axis of the plot is $2 R_{i}$, that is, the distance between the centers of the person and the robot, and the vertical axis is the interval $[0-1]$, where 1 is the best performance. Then, we penalise with 0 performance if $2 R_{i}<0.75 \mathrm{~m}$ or if $2 R_{i}>$ $3 \mathrm{~m}$. For the performance in angle, we have considered in the horizontal axis the difference between $\theta(t)-\theta_{r}$, where $\theta(t)$ is the angle between the couple human-robot that allows the joint navigation while both avoid the obstacles and $\theta_{r}$ is the actual angle between the couple human-robot. The best performance is for $0 \leq \theta(t)-\theta_{r} \leq 10$ degrees. If the difference is greater than 10 degrees, we penalize each increment of 10 degrees with -0.1 in performance.

In each of the simulation experiments, we evaluate both performances, distance in Fig. 5-left and angle in Fig. 5right. The results obtained for all the simulations show the 

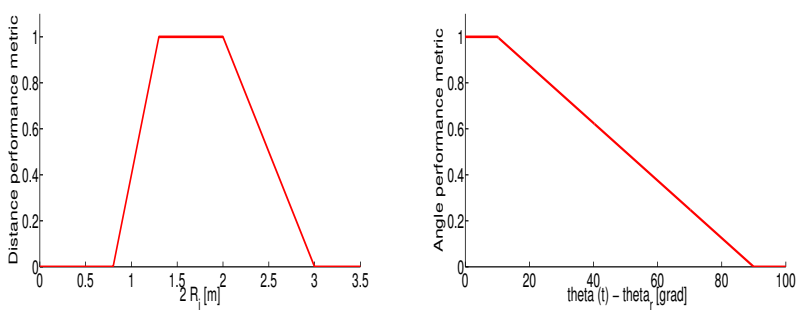

Fig. 4. The plot of the left shows the metric for the performance in distance and the plot of the right shows the metric for the performance in angle.

mean and the standard error of the performance. The results are very positive, since we have obtained a distance mean of 0.8996 and an angle mean of 0.9933 . The standard errors are 0.0155 for distance and $9.4694 e^{-4}$ for the angle.

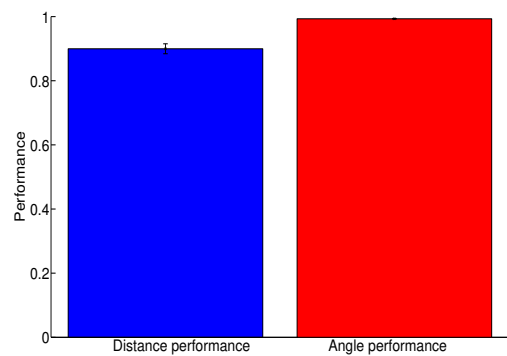

Fig. 5. The plot shows the mean and the standard error of the performance in distance and angle of the companion task of all the simulated experiments.

Furthermore, we include a plot where we compare the mean of the real angle and the desired angle of the companion along different experiments which can be seen in Fig. 6, Fig. 7 and Fig. 8. For the case of static obstacles, we can compare these angles for all the experiments, because is a static environment and the robot needs to achieve the same desired angle to accomplish the companion task throughout the experiment. In the case of the dynamic obstacles, we made the same comparisons, but only in the $4.7 \%$ of the experiments. In these $4.7 \%$ of the experiments, we consider a velocity of $0.3 \mathrm{~m} / \mathrm{s}$ for all the people of the environment. The same velocity is to obtain the same changes in angles at the same instant of time, and this velocity can be up of 0.3 $\mathrm{m} / \mathrm{s}$. In these experiments, we obtained the same differences between the two angles. Finally, we have a combination of both results in the case that we combine static and dynamic obstacles in the task.

\section{REAL-LIFE EXPERIMENTS}

The proposed method was also tested in real-live experiments with the Tibi robot of our institute. We used a controlled environment with a limited number of people, in a similar configuration of the simulation tests ( see Fig. 9). The location to do the experiments has been the FME (Facultat de Matemàtiques i Estadística) lab, an outdoor urban environment located at the South Campus of the Universitat Politècnica de Catalunya (UPC). We made 22 real-live experiments with 10 volunteers and no instructions were given to them. The volunteers were Master students, Phd and technicians. Their ages were between 21 and 35 years old. In these experiments, we evaluated the method in

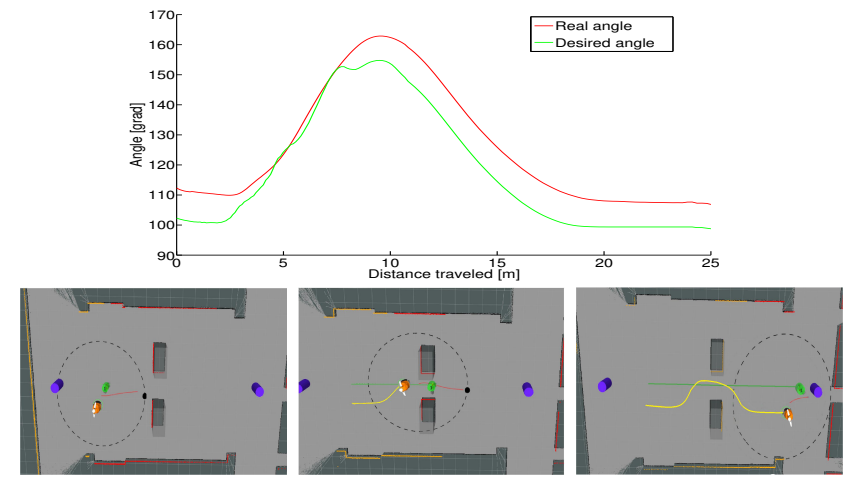

Fig. 6. The plot of the top shows the comparison between the real and desired angle of the couple. The plot of the bottom shows the simulated experiment for the case of joint static obstacle avoidance. The obstacles were the two squares of the center, the companion person was the green cylinder of id 1 and is inside the black circle with the robot in orange. The goals were the purple cylinders. The robot path is the yellow line and the companion person path is the green line.

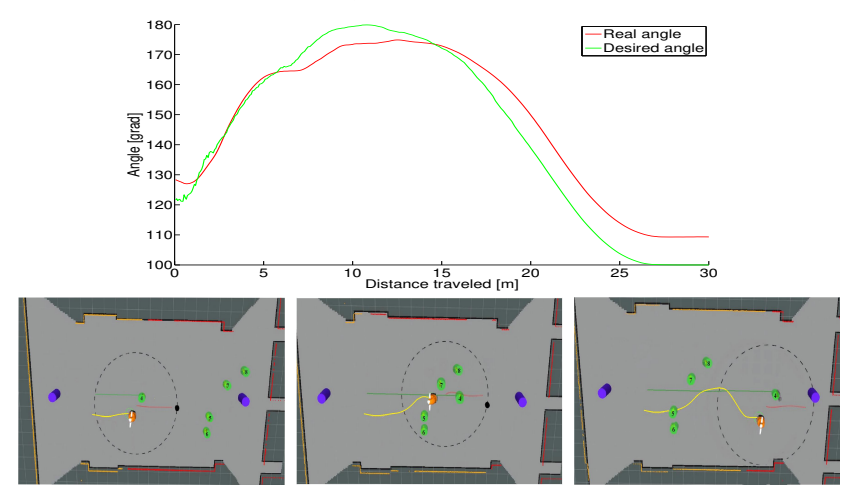

Fig. 7. The plot of the top shows the comparison between the real and desired angle for the couple. The plot of the bottom shows the simulated experiment for the case of joint dynamic obstacle avoidance. The companion person was the green cylinder of id 4 , which is inside the black circle with the orange robot, and the other green cylinders were the two people groups. The goals were the purple cylinders. The robot path is the yellow line and the companion person path is the green line.

a quantitative way using the same performance metrics that were used in simulation. The real-live experiments are shown in Fig. 9 and results in distance and angle performance can be seen in Fig. 10. Positive results were obtained. Regarding to the performance in distance we obtained a mean of 0.9115 and a standard error of 0.0094 , and in the angle performance, we obtained a mean of 0.7348 and a standard error of 0.0178 .

We added a link where you can see the video of the results of this work, https://youtu.be/4nKSdLjVu-c.

\section{CONCLUSIONS AND FUTURE WORK}

We have presented an adaptive human-robot side-by-side navigation approach based on Kinodynamic Anticipate Planning and Extended Social forces Model. Our model extends the work [8] to allow side-by-side accompany of a person by a robot in a dynamic environment.

The major contributions of this paper are twofold. First, we obtain the best path to go to the goal while accompanying a person in dynamic and static narrow passageways, by using a new cost function that takes into account the geometrical configuration between the human and the robot. The best path is obtained by minimizing all the cost functions in 


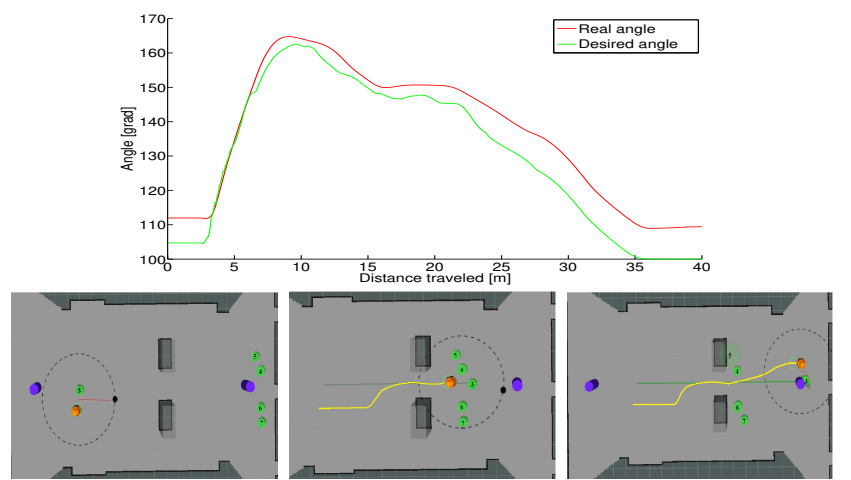

Fig. 8. The plot of the top shows the comparison between the real and desired angle for the couple. The plot of the bottom shows the simulated experiment for the case of joint dynamic and static obstacle avoidance. The companion person was the green cylinder of id 3, which is inside the black circle with the orange robot. The goals were the purple cylinders. The robot path is the yellow line and the companion person path is the green line.

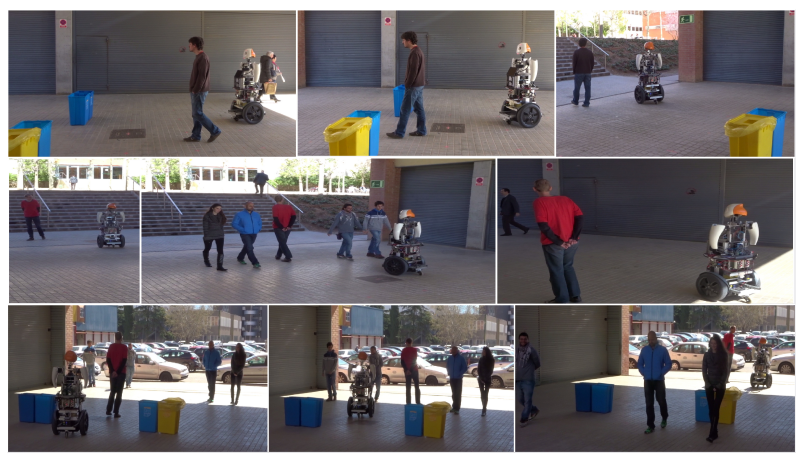

Fig. 9. The first three images show the case of static obstacle avoidance; the second three images show the case of dynamic obstacle avoidance; and the three last images show the case of dynamic and static obstacle avoidance.

a RRT planner. Second, we have redefined the Extended Social Force Model [8] to include a social force that tries to maintain the best geometrical configuration for the sideby-side accompany task while the couple follows the best path found. The result is a path where the geometrical configuration (distance and angle) between the human and the robot, adapts to the different static (due to obstacles) and dynamic (due to moving people) narrow passageways. The computation is done on-line and in real time. The new model has been tested in simulation and real-live experiments.

In future work, we will perform a qualitative analysis with non-trained volunteers. Also, we will ask people which path considers best to go to the final destination. Furthermore, we will intend to include a dependence between distance and angle performance and human walking speed.

\section{REFERENCES}

[1] M. A. Yousuf, Y. Kobayashi, Y. Kuno, A. Yamazaki, and K. Yamazaki, "Development of a mobile museum guide robot that can configure spatial formation with visitors," in International Conference on Intelligent Computing. Springer, 2012, pp. 423-432.

[2] Y. Chen, F. Wu, W. Shuai, N. Wang, R. Chen, and X. Chen, "Kejia robot-an attractive shopping mall guider," in International Conference on Social Robotics. Springer, 2015, pp. 145-154.

[3] A. Haasch, S. Hohenner, S. Hüwel, M. Kleinehagenbrock, S. Lang, I. Toptsis, G. A. Fink, J. Fritsch, B. Wrede, and G. Sagerer, "Bironthe bielefeld robot companion," in Proc. Int. Workshop on Advances in Service Robotics. Citeseer, 2004, pp. 27-32.

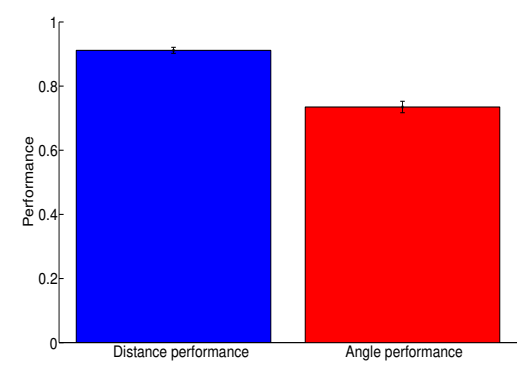

Fig. 10. The plot shows the performance in distance and in angle for all the real-live experiments, while the couple avoid static and dynamic obstacles at the same time.

[4] B. Graf, J. H. Wandosell, and C. Schaeffer, "Flexible path planning for nonholonomic mobile robots," in Proc. 4th European workshop on advanced Mobile Robots (EUROBOT01)., Fraunhofer Inst. Manufact. Eng. Automat.(IPS). Lund, Sweden, 2001, pp. 199-206.

[5] G. Ferrer, A. Garrell, F. Herrero, and A. Sanfeliu, "Robot social-aware navigation framework to accompany people walking side-by-side," pp. $1-19,2016$.

[6] A. Garrell and A. Sanfeliu, "Cooperative social robots to accompany groups of people," The International Journal of Robotics Research, vol. 31, no. 13, pp. 1675-1701, 2012.

[7] G. Ferrer and A. Sanfeliu, "Proactive kinodynamic planning using the extended social force model and human motion prediction in urban environments," in IEEE/RSJ international conference on Intelligent robots and systems. IEEE, 2014, pp. 1730-1735.

[8] — "Multi-objective cost-to-go functions on robot navigation in dynamic environments," in IEEE/RSJ international conference on Intelligent robots and systems. IEEE, 2015, pp. 3824-3829.

[9] D. Helbing and P. Molnar, "Social force model for pedestrian dynamics," Physical review E, vol. 51, no. 5, p. 4282, 1995.

[10] E. Prassler, D. Bank, B. Kluge, and M. Hagele, "Key technologies in robot assistants: Motion coordination between a human and a mobile robot," Transactions on Control, Automation and Systems Engineering, vol. 4, no. 1, pp. 56-61, 2002.

[11] E. Sviestins, N. Mitsunaga, T. Kanda, H. Ishiguro, and N. Hagita, "Speed adaptation for a robot walking with a human," in $A C M / I E E E$ International Conference on Human-Robot Interaction. IEEE, 2007, pp. $349-356$.

[12] R. Murakami, L. Y. Morales Saiki, S. Satake, T. Kanda, and H. Ishiguro, "Destination unknown: walking side-by-side without knowing the goal," in Proceedings of the ACM/IEEE international conference on Human-robot interaction. ACM, 2014, pp. 471-478.

[13] L. Y. Morales Saiki, S. Satake, R. Huq, D. Glas, T. Kanda, and N. Hagita, "How do people walk side-by-side?: using a computational model of human behavior for a social robot," in Proceedings of the seventh anпиal ACM/IEEE international conference on Human-Robot Interaction. ACM, 2012, pp. 301-308.

[14] Y. Morales, T. Kanda, and N. Hagita, "Walking together: side by side walking model for an interacting robot," Journal of Human-Robot Interaction, vol. 3, no. 2, pp. 51-73, 2014.

[15] M. Kuderer, H. Kretzschmar, and W. Burgard, "Teaching mobile robots to cooperatively navigate in populated environments," in IEEE/RSJ International Conference on Intelligent Robots and Systems. IEEE, 2013, pp. 3138-3143.

[16] G. Ferrer and A. Sanfeliu, "Bayesian human motion intentionality prediction in urban environments," Pattern Recognition Letters, vol. 44, pp. 134-140, 2014.

[17] V. Vaquero, E. Repiso, A. Sanfeliu, J. Vissers, and M. Kwakkernaat, "Low cost, robust and real time system for detecting and tracking moving objects to automate cargo handling in port terminals," in Robo 2015: Second Iberian Robotics Conference. Springer, 2016, pp. 491502.

[18] S. LaValle and J. Kuffner, "Randomized kinodynamic planning," The International Journal on Robotics Research, vol. 20, no. 5, pp. 378400, 2001. 